\title{
HOMOLOGICAL ACTION OF THE MODULAR GROUP ON SOME CUBIC MODULI SPACES
}

\author{
Wiliam M. Goldman and Walter D. Neumann
}

\begin{abstract}
We describe the action of the automorphism group of the complex cubic $x^{2}+y^{2}+z^{2}-x y z-2$ on the homology of its fibers. This action includes the action of the mapping class group of a punctured torus on the subvarieties of its $\operatorname{SL}(2, \mathbb{C})$ character variety given by fixing the trace of the peripheral element (so-called "relative character varieties"). This mapping class group is isomorphic to $\operatorname{PGL}(2, \mathbb{Z})$. We also describe the corresponding mapping class group action for the four-holed sphere and its relative $\mathrm{SL}(2, \mathbb{C})$ character varieties, which are fibers of deformations $x^{2}+y^{2}+z^{2}-x y z-2-P x-Q y-R z$ of the above cubic. The 2-congruence subgroup $\operatorname{PGL}(2, \mathbb{Z})_{(2)}$ still acts on these cubics and is the full automorphism group when $P, Q, R$ are distinct.
\end{abstract}

\section{Introduction}

Several important moduli spaces are described by cubic surfaces in affine space. The symmetries of the original moduli problem determines a group of transformations of the moduli space which generates a dynamical system. For two bounded surfaces $M$ - the one-holed torus and the four-holed sphere the relative $\operatorname{SL}(2, \mathbb{C})$-character varieties are described by cubic surfaces $\mathcal{M}$ in $\mathbb{C}^{3}$ of the form

$$
x^{2}+y^{2}+z^{2}-x y z=P x+Q y+R z+S
$$

upon which the mapping class group $\pi_{0} \operatorname{Homeo}(M, \partial M)$ acts by polynomial automorphisms. For these surfaces $\pi_{0} \operatorname{Homeo}(M, \partial M)$ is essentially the modular group $\mathrm{PGL}(2, \mathbb{Z})$. We compute the homology of $\mathcal{M}$ and its intersection form, and the resulting action of $\mathrm{PGL}(2, \mathbb{Z})$ on $H_{*}(\mathcal{M})$.

A relative $\operatorname{SL}(2, \mathbb{C})$-character variety of a compact bounded surface $M$ classifies equivalence classes of representations

$$
\rho: \pi_{1}(M) \longrightarrow \mathrm{SL}(2, \mathbb{C})
$$

with certain boundary conditions. Namely let $\partial_{i} M$ for $i=1, \ldots, n$ denote the boundary components of $M$ and choose $\delta_{i} \in \pi_{1}(M)$ corresponding to generators of $\pi_{1}\left(\partial_{i} M\right)$ respectively and and $t=\left(t_{1}, \ldots, t_{n}\right) \in \mathbb{C}^{n}$. Then the relative

Received by the editors February 4, 2004.

2000 Mathematics Subject Classification. 57M05, 30F60, 20H10.

Research supported under NSF grant DMS-0103889.

Research supported under NSF grants DMS-0083097 and DMS-0206464. 
$\mathrm{SL}(2, \mathbb{C})$-character variety with boundary data $t$ is the moduli space of representations $\rho$ where

$$
\operatorname{tr}\left(\rho\left(\delta_{i}\right)\right)=t_{i}
$$

for $i=1, \ldots, N$.

Suppose first that $M$ is a one-holed torus. Its fundamental group $\pi$ is free of rank two, and admits a redundant geometric presentation

$$
\left\langle X, Y, \delta_{1} \mid \delta_{1}=X Y X^{-1} Y^{-1}\right\rangle
$$

where $\delta_{1}$ corresponds to a generator of $\pi_{1}(\partial M)$. Fricke [5] proved that the character variety is an affine space $\mathbb{C}^{3}$ with coordinates

$$
\begin{aligned}
& x=\operatorname{tr}(\rho(X)) \\
& y=\operatorname{tr}(\rho(Y)) \\
& z=\operatorname{tr}(\rho(Z)), \quad Z=X Y .
\end{aligned}
$$

The resulting $\pi_{0}$ Homeo $(M, \partial M)$-action on this $\mathbb{C}^{3}$ factors through an action of $\operatorname{PGL}(2, \mathbb{Z})$. Namely, by Dehn (unpublished) and Nielsen [13], $\pi_{0} \operatorname{Homeo}(M, \partial M)$ $\cong \operatorname{Out}(\pi) \cong \mathrm{GL}(2, \mathbb{Z})$ via the action on the abelianization $\mathbb{Z}^{2}$ of $\pi=\pi_{1}(M)$. The center $\{ \pm I\}$ of $\mathrm{GL}(2, \mathbb{Z})$ is represented by the automorphism $X \mapsto X^{-1}, Y \mapsto$ $Y^{-1}, Z \mapsto Y Z^{-1} Y^{-1}$, which preserves traces and hence acts trivially on $\mathbb{C}^{3}$, so the action can be considered as an action of $\mathrm{GL}(2, \mathbb{Z}) /\{ \pm I\}=\mathrm{PGL}(2, \mathbb{Z})$.

The relative character varieties result by fixing the trace of $\rho\left(\delta_{1}\right)$, which is given by the polynomial

$$
\operatorname{tr}\left(\rho\left(X Y X^{-1} Y^{-1}\right)\right)=\kappa(\operatorname{tr}(\rho(X)), \operatorname{tr}(\rho(Y)), \operatorname{tr}(\rho(X Y))
$$

where

$$
\kappa(x, y, z):=x^{2}+y^{2}+z^{2}-x y z-2 .
$$

Thus, the action of $\operatorname{PGL}(2, \mathbb{Z})$ on $\mathbb{C}^{3}$ preserves this polynomial $\kappa(x, y, z)$.

The full automorphism group $\Gamma=\operatorname{Aut}(\kappa)$ of this polynomial $\kappa$ is generated by $\operatorname{PGL}(2, \mathbb{Z})$ and the "group of sign changes," namely the Klein 4-group $\Sigma \cong$ $C_{2} \times C_{2}$ that acts on $\mathbb{C}^{3}$ by $(x, y, z) \mapsto( \pm x, \pm y, \pm z)$ with an even number of minus signs. In fact (see Horowitz [9]) $\Gamma$ is the split extension:

$$
\Gamma=\operatorname{PGL}(2, \mathbb{Z}) \ltimes \Sigma .
$$

The dynamic behavior of the action of $\Gamma$ on the real character variety $\mathbb{R}^{3} \subset \mathbb{C}^{3}$ was described in [7], but little is known about the full action on $\mathbb{C}^{3}$. In this note we investigate the algebraic topology of this action of $\Gamma$ (and hence of $\operatorname{PGL}(2, \mathbb{Z})$ ) on the fibers of the polynomial $\kappa$.

The results can be summarized:

Theorem 1. The fibers $V_{t}:=\kappa^{-1}(t)$ of the polynomial $\kappa: \mathbb{C}^{3} \rightarrow \mathbb{C}$ have reduced homology only in dimension 2. There are two special fibers: $V_{2}$ and $V_{-2}$ with homology $H_{2}\left(V_{2}\right) \cong \mathbb{Z}$ and $H_{2}\left(V_{-2}\right) \cong \mathbb{Z}^{4}$, respectively. The generic fiber $V_{t}$ with $t \neq \pm 2$ has homology

$$
H_{2}\left(V_{t}\right)=H_{2}\left(V_{-2}\right) \oplus H_{2}\left(V_{2}\right) \cong \mathbb{Z}^{5} .
$$


The automorphism group $\Gamma$ acts on these homology groups via a homomorphism to $S_{4} \times C_{2}$, with the symmetric group $S_{4}$ acting by $\left(\rho_{4}\right) \oplus 1$ on $\mathbb{Z}^{4} \oplus \mathbb{Z}$, where $\rho_{4}$ $i s$ the standard permutation representation of $S_{4}$ on $\mathbb{Z}^{4}$, and the cyclic group $C_{2}$ acting by multiplication by \pm 1 on $\mathbb{Z}^{5}$.

The mapping class group Out $(\pi)$, acting on $\mathbb{C}^{3}$ as the subgroup $\operatorname{PGL}(2, \mathbb{Z})$ of $\Gamma$, acts on the above homology groups as the subgroup $S_{3} \times C_{2}$ of $S_{4} \times C_{2}$, where the $S_{3}$ is the subgroup of $S_{4}$ that fixes one of the generators of $\mathbb{Z}^{4}$.

The homomorphism of $\Gamma$ to $S_{4} \times C_{2}$ is as follows: The homomorphism to $C_{2}$ is the determinant map

$$
\Gamma=\mathrm{PGL}(2, \mathbb{Z}) \ltimes \Sigma \rightarrow \mathrm{PGL}(2, \mathbb{Z}) \stackrel{\text { det }}{\longrightarrow} C_{2}
$$

The homomorphism to $S_{4}$ arises via reduction modulo 2 :

$$
\Gamma=\mathrm{PGL}(2, \mathbb{Z}) \ltimes \Sigma \longrightarrow \mathrm{PGL}(2, \mathbb{Z} / 2) \ltimes \Sigma=S_{3} \ltimes \Sigma \cong S_{4} .
$$

Here $\mathrm{PGL}(2, \mathbb{Z} / 2) \ltimes \Sigma \cong S_{4}$ because $\mathrm{PGL}(2, \mathbb{Z} / 2) \ltimes \Sigma$ is the group of affine automorphisms of an affine plane $\mathbb{A}=\mathbb{A}^{2}(\mathbb{Z} / 2)$ and every permutation of the 4 element set $\mathbb{A}$ is affine. The subgroup $\Sigma$ is the group of translations, which acts simply transitively on $\mathbb{A}$. The group $S_{3}$ that fixes a chosen origin in $\mathbb{A}$ and permutes the other three points is the group of linear automorphisms $\operatorname{PGL}(2, \mathbb{Z} / 2)=G L(2, \mathbb{Z} / 2)$ (since $\mathbb{Z} / 2$ contains only one nonzero scalar,

$$
\operatorname{PGL}(2, \mathbb{Z} / 2)=\mathrm{GL}(2, \mathbb{Z} / 2)=\operatorname{PSL}(2, \mathbb{Z} / 2)=\operatorname{SL}(2, \mathbb{Z} / 2)) \text {. }
$$

In terms of the punctured torus $M, \mathbb{A}$ can be identified with the set of spin structures on a trivial vector bundle; specifically its vector space of translations identifies with the cohomology group $H^{1}(M, \mathbb{Z} / 2)$.

Note that the kernel of $\Gamma=\operatorname{PGL}(2, \mathbb{Z}) \ltimes \Sigma \longrightarrow \mathrm{PGL}(\mathbb{Z} / 2,2) \ltimes \Sigma=S_{4}$ is the congruence subgroup

$$
\operatorname{PGL}(2, \mathbb{Z})_{(2)}:=\left\{\left(\begin{array}{ll}
a & b \\
c & d
\end{array}\right) \in \operatorname{PGL}(2, \mathbb{Z}) \mid b \equiv c \equiv 0 \bmod 2\right\}
$$

of $\operatorname{PGL}(2, \mathbb{Z})$. We have a commutative diagram with exact rows and columns

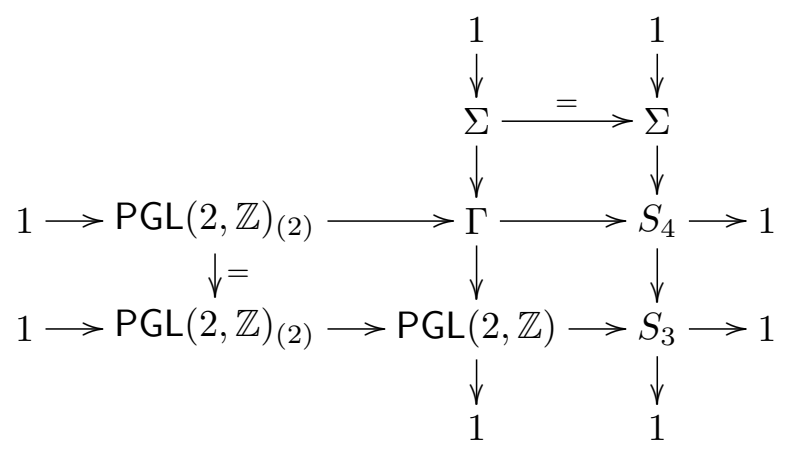

The right-hand column identifies with the short exact sequence

$$
1 \longrightarrow \mathbb{Z} / 2 \oplus \mathbb{Z} / 2 \longrightarrow \operatorname{Aff}(2, \mathbb{Z} / 2) \longrightarrow \mathrm{PGL}(2, \mathbb{Z} / 2) \longrightarrow 1
$$


All four short exact sequences of the diagram are split exact. In particular,

$$
\Gamma \cong \mathrm{PGL}(2, \mathbb{Z})_{(2)} \rtimes S_{4} .
$$

We now describe the case of the four holed sphere $N$. The group $\Gamma$ arises in a new context in this discussion, as the mapping class group $\pi_{0} \operatorname{Homeo}(N, \partial N)$. The relative $\mathrm{SL}(2, \mathbb{C})$ character varieties for $N$ are again certain cubic surfaces. We first discuss the automorphisms of these cubic surfaces.

Theorem 2. For any $P, Q, R \in \mathbb{C}$, the action of $\mathrm{PGL}(2, \mathbb{Z})_{(2)}$ on $\mathbb{C}^{3}$ that preserves the polynomial $\kappa$ deforms to an action that preserves the cubic polynomial

$$
\kappa_{P, Q, R}(x, y, z):=x^{2}+y^{2}+z^{2}-x y z-P x-Q y-R z-2 .
$$

Each nonsingular fiber $V$ of $\kappa_{P, Q, R}$ is diffeomorphic to a non-singular fiber of $\kappa$, so it still has homology $\mathrm{H}_{2}(V) \cong \mathbb{Z}^{5}$, while a singular fiber has homology a quotient of this. The action of $\mathrm{PGL}(2, \mathbb{Z})_{(2)}$ on the homology of fibers of $\kappa_{P, Q, R}$ is again by multiplication by \pm 1 via the determinant homomorphism $\mathrm{PGL}(2, \mathbb{Z})_{(2)} \rightarrow C_{2}=\{ \pm 1\}$.

$\mathrm{PGL}(2, \mathbb{Z})_{(2)}$ is the full automorphism group of $\kappa_{P, Q, R}$ if $P, Q, R$ are distinct, while otherwise the full automorphism group is $\mathrm{PGL}(2, \mathbb{Z})_{(2)}$ extended by an appropriate subgroup of $S_{4}$ (those permutations and sign changes of $x, y, z$ that preserve $\kappa_{P, Q, R}$; - if at most one of $P, Q, R$ is zero then there are only permutations).

Each relative character variety for $N$ is a fiber of some cubic polynomial $\kappa_{P, Q, R}(x, y, z)$. The mapping class group that fixes all four boundary components satisfies

$$
\pi_{0} \operatorname{Homeo}\left(N, \partial_{1} N, \ldots, \partial_{4} N\right) \cong \operatorname{PGL}(2, \mathbb{Z})_{(2)},
$$

and its action on the relative character variety is by the action of the above theorem.

If the trace constraints at some boundary components of $M$ are identical, then the relevant mapping class group is $\operatorname{PGL}(2, \mathbb{Z})_{(2)} \rtimes K$ where $K \subset S_{4}$ is the group of permutations that preserve trace constraints. Its action on the relative character variety is by the quotient $\operatorname{PGL}(2, \mathbb{Z})_{(2)} \rtimes \pi(K)$ where $\pi: S_{4} \rightarrow$ $S_{3}$. In particular, if the trace constraints at all four boundary components of $N$ are equal, then the relevant mapping class group is a semidirect product $\operatorname{PGL}(2, \mathbb{Z})_{(2)} \rtimes S_{4}$. In fact it is the same semidirect product as that of equation $(2)$, so

$$
\pi_{0} \operatorname{Homeo}(N, \partial N) \cong \Gamma \text {. }
$$

But its action on $\mathbb{C}^{3}$ in Theorem 2 is via its homomorphism to $\operatorname{PGL}(2, \mathbb{Z})$, with kernel $\Sigma$, while the action of $\Gamma$ on $\mathbb{C}^{3}$ of Theorem 1 was faithful.

We also determine the intersection form on homology of fibers:

Proposition 3. The intersection form on the homology $H_{2}\left(V_{t}\right)$ of a general fiber of any one of the cubics $\kappa_{P, Q, R}$ with respect to the basis used in the above 
theorems is

$$
\left(\begin{array}{ccccc}
-2 & 0 & 0 & 0 & 1 \\
0 & -2 & 0 & 0 & 1 \\
0 & 0 & -2 & 0 & 1 \\
0 & 0 & 0 & -2 & 1 \\
1 & 1 & 1 & 1 & -2
\end{array}\right)
$$

Acknowledgments. Goldman is grateful to J. Damon, L. Ein, H. King, A. Libgober and J. W. Wood for valuable conversations.

\section{Vanishing cycles and homology}

It is known (e.g., [12]) that the homology of a general fiber of a polynomial map $\kappa$ comes from vanishing cycles of the singularities and vanishing cycles "at infinity."

Broughton [2] shows that a polynomial has no vanishing cycles at infinity if it is "tame", that is, $|d \kappa|$ is bounded away from zero outside some compact set. Our polynomial $\kappa$ is tame, in fact:

Lemma 1.1. $|d \kappa|$ tends to infinity as $|(x, y, z)| \rightarrow \infty$.

Proof.

$$
d \kappa=(2 x-y z) d x+(2 y-x z) d y+(2 z-x y) d z=: a d x+b d y+c d z
$$

We shall show that $\|(a, b, c)\|$ bounded implies $\|(x, y, z)\|$ bounded. Consider a sequence of points $(x, y, z)$ going to infinity for which $(a, b, c)$ stays bounded. If two coordinates, say $x$ and $y$, stay bounded then $c=2 z-x y$ tends to infinity, a contradiction. So at least two coordinates, say $y$ and $z$, must approach infinity in the sequence. But for $z \neq \pm 2$ the equations $2 x=a+y z$ and $2 y=b+x z$ imply

$$
y=\frac{2 b+a z}{4-z^{2}}
$$

which approaches 0 as $z \rightarrow \infty$, another contradiction.

For a tame polynomial, the homology of the general fiber is given by the vanishing cycles of singularities, so we need to find the singularities of $\kappa$ :

$$
2 x-y z=2 y-x z=2 z-x y=0
$$

implies the critical points of $\kappa$ are:

$$
(2,-2,-2),(-2,2,-2),(-2,-2,2),(2,2,2),(0,0,0) \text {. }
$$

The first four singular points are on the fiber $V_{2}$ while the singular point $(0,0,0)$ is on $V_{-2}$. For any one of the singular points $\left(2 \epsilon_{1}, 2 \epsilon_{2}, 2 \epsilon_{1} \epsilon_{2}\right)$ with $\epsilon_{i}= \pm 1$ we introduce local coordinates

$$
\hat{x}=\epsilon_{1} x-2, \quad \hat{y}=\epsilon_{2} y-2, \quad \hat{z}=\epsilon_{1} \epsilon_{2} z-2 .
$$

In these local coordinates we have

$$
\kappa(x, y, z)=\hat{x}^{2}+\hat{y}^{2}+\hat{z}^{2}-2 \hat{x} \hat{y}-2 \hat{x} \hat{z}-2 \hat{y} \hat{z}-\hat{x} \hat{y} \hat{z} .
$$


The Hessian at $(0,0,0)$ is $2 I$ and at each of the other four singular points (using the above local coordinates) it is

$$
2\left(\begin{array}{ccc}
1 & -1 & -1 \\
-1 & 1 & -1 \\
-1 & -1 & 1
\end{array}\right)
$$

Since these Hessians are non-singular, we have a quadratic singularity at each point, so each singular point contributes a single vanishing cycle to the homology of other fibers of $\kappa$. We hence have:

$$
\begin{aligned}
H_{2}\left(V_{2}\right) \cong \mathbb{Z} \quad & \text { generated by the vanishing cycle at }(0,0,0) \\
H_{2}\left(V_{-2}\right) \cong \mathbb{Z}^{4} & \text { generated by the vanishing cycles at }( \pm 2, \pm 2, \pm 2) \\
H_{2}\left(V_{t}\right) \cong \mathbb{Z}^{5} & \text { otherwise, generated by all five vanishing cycles }
\end{aligned}
$$

The vanishing cycle at a quadratic singularity is only defined up to sign; a self-isomorphism of the singularity whose derivative has negative determinant multiplies the cycle by -1 . We will choose a basis of $\mathbb{Z}^{5}=\mathbb{Z}^{4} \oplus \mathbb{Z}$ by choosing vanishing cycles at $(2,-2,-2)$ and $(0,0,0)$ and using the identity map between our chosen local coordinates $\hat{x}, \hat{y}, \hat{z}$ at the four singular points $( \pm 1, \pm 1, \pm 1)$ to choose the vanishing cycles at the other three singular points. We will order our basis according to the ordering of singular points in (3) above.

\section{Action of $\Gamma$}

The Klein 4-group $\Sigma$ of sign-changes permutes the first four singular points (3) of $\kappa$ by the regular permutation representation. It acts as the identity map in the local coordinates that we introduced above. It therefore simply permutes the four vanishing cycles corresponding to these points. Moreover, it fixes the singular point $(0,0,0)$ and acts with positive determinant in local coordinates there, so it fixes the corresponding vanishing cycle. It hence acts on $\mathbb{Z}^{5}$ by the 4-group whose non-trivial elements are:

$$
\left(\begin{array}{lllll}
0 & 1 & 0 & 0 & 0 \\
1 & 0 & 0 & 0 & 0 \\
0 & 0 & 0 & 1 & 0 \\
0 & 0 & 1 & 0 & 0 \\
0 & 0 & 0 & 0 & 1
\end{array}\right), \quad\left(\begin{array}{lllll}
0 & 0 & 1 & 0 & 0 \\
0 & 0 & 0 & 1 & 0 \\
1 & 0 & 0 & 0 & 0 \\
0 & 1 & 0 & 0 & 0 \\
0 & 0 & 0 & 0 & 1
\end{array}\right), \quad\left(\begin{array}{lllll}
0 & 0 & 0 & 1 & 0 \\
0 & 0 & 1 & 0 & 0 \\
0 & 1 & 0 & 0 & 0 \\
1 & 0 & 0 & 0 & 0 \\
0 & 0 & 0 & 0 & 1
\end{array}\right) .
$$

The group $\operatorname{PGL}(2, \mathbb{Z})$ is generated by $\operatorname{PSL}(2, \mathbb{Z})$ and the involution $\left(\begin{array}{rr}-1 & 0 \\ 0 & 1\end{array}\right)$. We first discuss the group $\operatorname{PSL}(2, \mathbb{Z})$. It is the free product of cyclic groups of orders 2 and 3 generated by

$$
\left(\begin{array}{cc}
0 & -1 \\
1 & 0
\end{array}\right) \text { and }\left(\begin{array}{cc}
1 & -1 \\
1 & 0
\end{array}\right)
$$


respectively. These are represented by automorphisms

$$
\begin{aligned}
\alpha: & X \mapsto Y, \quad Y \mapsto X^{-1} \\
\beta: & X \mapsto X Y, \quad Y \mapsto X^{-1} .
\end{aligned}
$$

Recalling that an automorphism $\gamma$ of $\pi$ acts on characters by $(\gamma \chi)(g)=$ $\chi \circ \gamma^{-1}(g)$, the action of the above two automorphisms on characters are:

$$
\begin{array}{rlrl}
\alpha_{*}: & & (x, y, z) & \mapsto(y, x, x y-z) \\
\beta_{*}: & (x, y, z) \mapsto(y, z, x)
\end{array}
$$

respectively.

The map $\alpha_{*}$ exchanges the first two singular points and fixes the other three. Its derivative acts with determinant 1 in the local coordinates at the singular points. It thus acts on the homology $\mathbb{Z}^{5}$ of a general fiber by:

$$
\left(\begin{array}{lllll}
0 & 1 & 0 & 0 & 0 \\
1 & 0 & 0 & 0 & 0 \\
0 & 0 & 1 & 0 & 0 \\
0 & 0 & 0 & 1 & 0 \\
0 & 0 & 0 & 0 & 1
\end{array}\right)
$$

The map $\beta_{*}$ of $\mathbb{C}^{3}$ permutes the first three singular points of (3) above and fixes the other two. It also acts with determinant 1 in the local coordinates at the singular points. It thus acts on the homology $\mathbb{Z}^{5}$ of a general fiber by:

$$
\left(\begin{array}{lllll}
0 & 0 & 1 & 0 & 0 \\
1 & 0 & 0 & 0 & 0 \\
0 & 1 & 0 & 0 & 0 \\
0 & 0 & 0 & 1 & 0 \\
0 & 0 & 0 & 0 & 1
\end{array}\right)
$$

The Klein 4-group $\Sigma$ and the elements $\alpha$ and $\beta$ generate the permutation representation of $S_{4}$ on the first four coordinates of $\mathbb{Z}^{5}$, as promised in the theorem of the Introduction.

Finally we discuss the involution

$$
\left(\begin{array}{cc}
-1 & 0 \\
0 & 1
\end{array}\right) \in \operatorname{PGL}(2, \mathbb{Z})
$$

The automorphism of $\pi$ defined by

$$
\gamma: X \mapsto X^{-1}, Y \mapsto Y
$$

represents this involution, and induces the map

$$
\gamma_{*}:(x, y, z) \mapsto(x, y, x y-z)
$$

on the character variety $\mathbb{C}^{3}$. This fixes all the singular points of $\kappa$ and its derivative acts with determinant -1 in the local coordinates at each point. It 
thus reverses the signs of all the vanishing cycles, so it acts on $\mathbb{Z}^{5}$ by the matrix

$$
\left(\begin{array}{ccccc}
-1 & 0 & 0 & 0 & 0 \\
0 & -1 & 0 & 0 & 0 \\
0 & 0 & -1 & 0 & 0 \\
0 & 0 & 0 & -1 & 0 \\
0 & 0 & 0 & 0 & -1
\end{array}\right) .
$$

\section{Intersection form}

Consider a general fiber $V_{t}$ (that is, $t \neq \pm 2$ ). It is well known that the vanishing cycle of a quadratic singularity has self-intersection number -2 . The vanishing cycles arising from the four singularities on $V_{2}$ are clearly disjoint. Thus the intersection form on $H_{2}\left(V_{t}\right)$ with respect to our basis is

$$
\left(\begin{array}{ccccc}
-2 & 0 & 0 & 0 & a_{1} \\
0 & -2 & 0 & 0 & a_{2} \\
0 & 0 & -2 & 0 & a_{3} \\
0 & 0 & 0 & -2 & a_{4} \\
a_{1} & a_{2} & a_{3} & a_{4} & -2
\end{array}\right)
$$

where $a_{1}, \ldots, a_{4}$ are still to be determined. Since an index 2 subgroup of $\Gamma$ permutes the first four basis elements, $a_{1}=a_{2}=a_{3}=a_{4}$. Call this common value $a$. We must show $a= \pm 1$ (the sign depends on how we have oriented the vanishing cycles over $t=2$ and $t=-2$ with respect to each other, and is thus indeterminate).

The intersection matrix has determinant $32\left(1-a^{2}\right)$. Let $\hat{V}_{t}$ be the intersection of $V_{t}$ with a very large ball around the origin and $M^{3}=\partial \hat{V}_{t}$ the "link at infinity" of $V_{t}$. Then $V_{t}$ retracts to $\hat{V}_{t}$, so they have the same homology and intersection form. The homology exact sequence

$$
H_{2}\left(\hat{V}_{t}\right) \rightarrow H_{2}\left(\hat{V}_{t}, M^{3}\right) \rightarrow H_{1}\left(M^{3}\right) \rightarrow 0
$$

and Poincaré-Lefschetz duality

$$
\left.H_{2}\left(\hat{V}_{t}, M^{3}\right) \cong H^{2}\left(\hat{V}_{t}\right)\right) \cong H_{2}\left(\hat{V}_{t}\right)^{*}
$$

yield a standard long exact sequence

$$
H_{2}\left(\hat{V}_{t}\right) \rightarrow H_{2}\left(\hat{V}_{t}\right)^{*} \rightarrow H_{1}\left(M^{3}\right) \rightarrow 0
$$

where the first arrow is given by intersection form. Thus $H_{1}\left(M^{3}\right)$ is the cokernel of the intersection matrix, so $a= \pm 1$ if and only if $H_{1}\left(M^{3}\right)$ is infinite (and otherwise $\left|H_{1}\left(M^{3}\right)\right|$ would equal $\left.\left.32\left|1-a^{2}\right|\right)\right)$. It thus remains to prove that $H_{1}\left(M^{3}\right)$ is indeed infinite.

Consider $M^{3}$ as a regular neighborhood boundary of the divisor at infinity of the closure $\bar{V}_{t}$ of $V_{t}$ in $\mathbb{P}^{3}(\mathbb{C})=\mathbb{C}^{3} \cup \mathbb{C P}^{2}$. Since the highest order term of $\kappa$ is $x y z, \bar{V}_{t}$ intersects the projective plane at infinity in the union of the three lines $x=0, y=0$, and $z=0$ (these are three of the 27 lines on a cubic surface; we will meet the other 24 lines on $V_{t}$ in the next section). It is easy to check 
in local coordinates that $\bar{V}_{t}$ is non-singular at infinity (and hence globally nonsingular except when $t= \pm 2$ ). The three lines at infinity of $V_{t}$ intersect in a cyclic configuration, so their neighborhood boundary certainly has infinite first homology, as desired.

In fact (see [11]), the neighborhood boundary $M^{3}$ of any cyclic configuration of rational curves in a non-singular complex surface is a $T^{2}$ bundle over $S^{1}$ whose monodromy can be computed from the self-intersection numbers $e_{i}$ of the curves as

$$
A=\prod_{i}\left(\begin{array}{cc}
0 & -1 \\
1 & -e_{i}
\end{array}\right) .
$$

In our case it is not hard to calculate that each of the three curves has selfintersection -1 (this is true for all 27 lines on a cubic surface) so the monodromy is

$$
\left(\begin{array}{cc}
0 & -1 \\
1 & 1
\end{array}\right)^{3}=\left(\begin{array}{cc}
-1 & 0 \\
0 & -1
\end{array}\right)
$$

and hence

$$
H_{1}\left(M^{3}\right)=\mathbb{Z} \oplus(\mathbb{Z} / 2)^{2} .
$$

One can check that this agrees with the cokernel of the intersection matrix of the proposition.

\section{Topology of fibers via the 27 lines on a cubic}

An alternative way of computing the homology of the fibers of $\kappa$ and the action of $\Gamma$ involves the projection to a coordinate line, and provides further insight into the topology. We sketch it briefly, hopefully with sufficient explanation that the reader can fill in the details. It is, in fact, the way we first obtained the results.

Suppose $t \neq \pm 2$ and consider the projection $\pi: V_{t} \rightarrow \mathbb{C}$ given by the $z$ coordinate. The general fiber $\pi^{-1}(s)$ of this projection is given by $x^{2}+y^{2}-x y s=$ $t+2-s^{2}$, which we can write as

$$
(x-\lambda y)\left(x-\lambda^{\prime} y\right)=t+2-s^{2}
$$

with $\lambda, \lambda^{\prime}=\frac{1}{2}\left(s \pm \sqrt{s^{2}-4}\right)$. Thus $\pi^{-1}(s) \cong \mathbb{C}^{*}$ by the map $(x, y) \mapsto x-\lambda y$ unless $\lambda=\lambda^{\prime}$ or $t+2-s^{2}=0$. The general fiber of $\pi$ is thus $\mathbb{C}^{*}$, while the special fibers occur for $s= \pm 2$ and $s= \pm \sqrt{t+2}$. These special fibers are:

$$
\begin{aligned}
\pi^{-1}(2)=L_{1} \cup L_{2} ; & L_{1}=\{x=y+\sqrt{t-2}\}, \\
L_{2} & =\{x=y-\sqrt{t-2}\} \\
\pi^{-1}(\sqrt{t+2})=L_{3} \cup L_{4} ; & L_{3}=\left\{\frac{x}{y}=\frac{\sqrt{t+2}+\sqrt{t-2}}{2}\right\}, \\
L_{4} & =\left\{\frac{x}{y}=\frac{\sqrt{t+2}-\sqrt{t-2}}{2}\right\}
\end{aligned}
$$




$$
\begin{aligned}
\pi^{-1}(-\sqrt{t+2})=L_{5} \cup L_{6} ; & L_{5}=\left\{\frac{x}{y}=-\frac{\sqrt{t+2}+\sqrt{t-2}}{2}\right\}, \\
L_{6} & =\left\{\frac{x}{y}=-\frac{\sqrt{t+2}-\sqrt{t-2}}{2}\right\} \\
\pi^{-1}(-2)=L_{7} \cup L_{8} ; & L_{7}=\{x=-y+\sqrt{t-2}\}, \\
L_{8} & =\{x=-y-\sqrt{t-2}\}
\end{aligned}
$$

These 8 lines $L_{1}, \ldots, L_{8}$ and the analogous lines coming from the projections to the $x$ and $y$ coordinates give 24 lines, which with the 3 lines at infinity give the full 27 lines on the projective cubic $\bar{V}_{t}$.

Choose small disks in $\mathbb{C}$

$$
D_{1}, D_{2}, D_{3}, D_{4} \text { around } z=2, \sqrt{t+2},-\sqrt{t+2},-2,
$$

and paths:

$$
\begin{aligned}
& \gamma_{1} \text { from } 2 \text { to } \sqrt{t+2}, \\
& \gamma_{2} \text { from } \sqrt{t+2} \text { to }-\sqrt{t+2}, \\
& \gamma_{3} \text { from }-\sqrt{t+2} \text { to }-2,
\end{aligned}
$$

so that these paths do not intersect each other except at endpoints.

Then $X:=\pi^{-1}\left(D_{1} \cup \gamma_{1} \cup D_{2} \cup \gamma_{2} \cup D_{3} \cup \gamma_{3} \cup D_{4}\right)$ is a deformation retract of $V_{t}$. Moreover

$$
\begin{array}{ll}
\pi^{-1}\left(D_{1}\right) \simeq S^{2} \simeq \pi^{-1}\left(D_{4}\right) & (\text { see below }) \\
\pi^{-1}\left(D_{2}\right) \simeq * \simeq \pi^{-1}\left(D_{3}\right) & \left(\text { since } \pi^{-1}( \pm \sqrt{t+2}) \simeq *\right)
\end{array}
$$

so a simple Mayer-Vietoris calculation of homology of $X$ gives

$$
\begin{array}{lrlllllllll}
H_{2}\left(V_{t}\right) \cong & \mathbb{Z} & \oplus & \mathbb{Z} & \oplus & \mathbb{Z} & \oplus & \mathbb{Z} & \oplus & \mathbb{Z} \\
\text { generators: } & \alpha_{1} & & \alpha_{2} & & \alpha_{3} & & \alpha_{4} & & \alpha_{5}
\end{array}
$$

where:

$$
\begin{array}{r}
\alpha_{1} \text { is a generator of } H_{2}\left(\pi^{-1}\left(D_{1}\right),\right. \\
\alpha_{5} \text { is a generator of } H_{2}\left(\pi^{-1}\left(D_{4}\right),\right. \\
\alpha_{2}, \alpha_{3}, \alpha_{4} \text { are supported over } \gamma_{1}, \gamma_{2}, \gamma_{3} .
\end{array}
$$

We shall see that the intersection form for this basis is

$$
\left(\begin{array}{ccccc}
-4 & 2 & 0 & 0 & 0 \\
2 & -2 & -1 & 0 & 0 \\
0 & -1 & -2 & 1 & 0 \\
0 & 0 & 1 & -2 & 2 \\
0 & 0 & 0 & 2 & -4
\end{array}\right)
$$

To describe the $\alpha_{i}$ more explicitly we draw the special fibers and $\pi^{-1}\left(D_{i}\right)$ in the projective completion $\bar{V}_{t}$ of $V_{t}$ : 


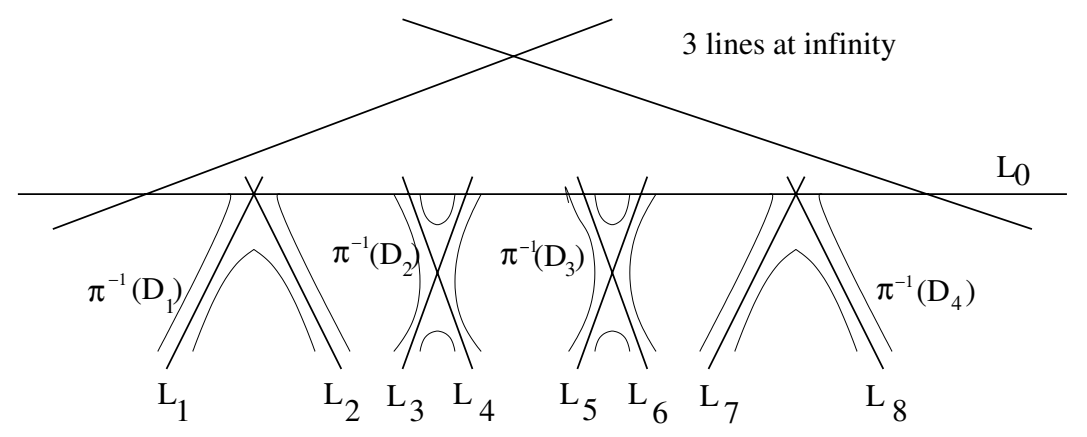

Each line in the diagram has self-intersection number -1 . We first describe the classes $\alpha_{2}, \alpha_{3}, \alpha_{4}$.

For $\alpha_{2}$, choose an arc $\gamma$ in the line $L_{0}$ at infinity from the point

$$
L_{0} \cap L_{2}=[1: 1]
$$

to the point

$$
L_{0} \cap L_{3}=\left[\frac{\sqrt{t+2}+\sqrt{t-2}}{2}: 1\right] .
$$

We can form the connected sum of $L_{2}$ and $-L_{3}$ by connecting them by a tube along this arc (the tube is $s^{-1}(\gamma)$ where $s: \partial N L_{0} \rightarrow L_{0}$ is the projection of the boundary of a tubular neighborhood of $L_{0}$ ). This connected sum $L_{2} \#-L_{3}$ lies in $V_{t}$ and represents the class $\alpha_{2}$.

We construct $\alpha_{3}$ and $\alpha_{4}$ similarly as $L_{4} \#-L_{5}$ and $L_{5} \#-L_{7}$. We shall see later that the choices of the tubes at infinity to form these connected sums does not affect the homology classes.

The classes $\alpha_{1}$ and $\alpha_{5}$ are constructed similarly as $L_{1} \#-L_{2}$ and $L_{7} \#-L_{8}$, but it is not so clear how the tubes are chosen. To clarify this blow up the point $L_{0} \cap L_{1}$;

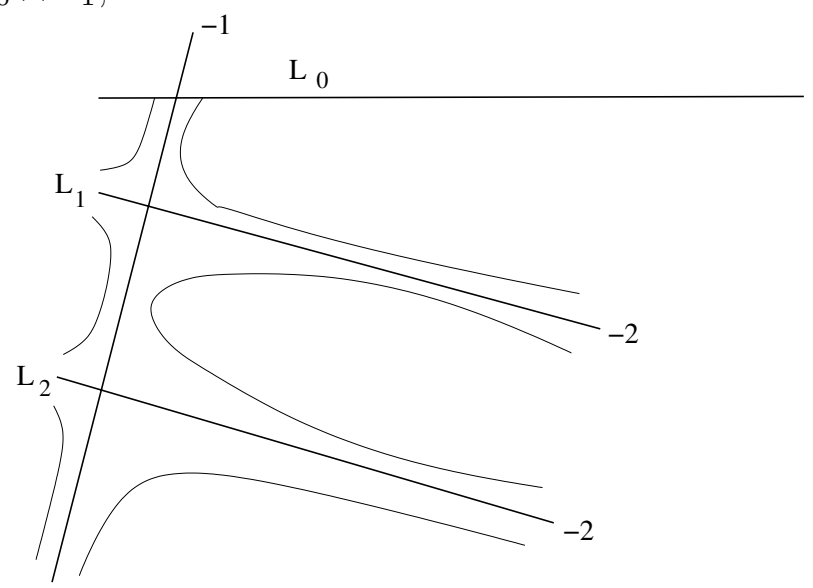

then $\pi^{-1}\left(D_{1}\right)$ can be seen as the union of a portion $N_{E}$ in a neighborhood of the exceptional curve $E$ and portions $N_{1}$ and $N_{2}$ in neighborhoods of $L_{1}, L_{2}$. $N_{E}$ is a $\left(D^{2}-\{0\}\right)$-bundle over $E-\left(E \cap L_{0}\right)$, hence homotopy equivalent to a circle. Adding $N_{1}$ and $N_{2}$ to this just adds 2-handles along parallel copies of the 
circle, so $\pi^{-1}\left(D_{1}\right) \simeq S^{2}$, as claimed earlier. The $S^{2}$ can be seen as $L_{1} \#-L_{2}$ with the connected sum formed along a tube over an arc in $E$. Its homology class $\alpha_{1}$ thus has self-intersection number

$$
\alpha_{1} \cdot \alpha_{1}=L_{1} \cdot L_{1}+L_{2} \cdot L_{2}=-2+(-2)=-4
$$

(since blowing up on $L_{1}$ and $L_{2}$ has decreased their self-intersection numbers by 1). The next paragraph gives an alternative argument.

We can see mutual intersection numbers of all our elements as follows. In the projective cubic $\bar{V}_{t}$ an element such as $\alpha_{2}=L_{2} \#-L_{3}$ is homologous to the sum $L_{2}+\left(-L_{3}\right)$, since the tube used to perform connected sum bounds a solid tube. Since the inclusion $V_{t} \rightarrow \bar{V}_{t}$ respects intersection number, we can simply compute intersection numbers in $\bar{V}_{t}$. Thus, for instance, $\alpha_{2}$ clearly has self-intersection number

$$
L_{2} \cdot L_{2}+L_{3} \cdot L_{3}=-2 \text {. }
$$

The claimed intersection matrix for the basis $\alpha_{1}, \ldots, \alpha_{5}$ is now easy to verify.

To see that the choices of the "tubes at infinity" do not affect the homology classes $\alpha_{2}, \alpha_{3}, \alpha_{4}$, note that if we move the path in $E_{0}$ defining such a tube past an intersection point $E_{0} \cap E_{i}$ then we change the homology class by the class of a torus $s^{-1}(\delta)$, where $\delta$ is a small loop in $E_{0}$ around this point. Such a torus is supported entirely in $\pi^{-1}\left(D_{i}\right)$ and has self-intersection number 0 , so its homology class is zero.

One can verify that the ordered basis of section 1 , corresponding to the vanishing cycles at

$$
(2,-2,-2), \quad(-2,2,-2), \quad(-2,-2,2), \quad(2,2,2), \quad(0,0,0),
$$

is

$$
-\left(\alpha_{4}+\alpha_{5}\right), \quad-\alpha_{4}, \quad \alpha_{1}+\alpha_{2}, \quad \alpha_{2}, \quad-\alpha_{3} .
$$

In terms of our lines these are:

$$
L_{8}-L_{5}, \quad L_{7}-L_{5}, \quad L_{1}-L_{3}, \quad L_{2}-L_{3}, \quad L_{5}-L_{4} .
$$

Recall that we only determined the basis of section 1 up to an overall sign. If we replace each $L_{i}$ by the other line in the same fiber of $\pi$, so $L_{8}-L_{5}$ becomes $L_{7}-L_{6}$ for example, each element of the basis is replaced by its negative.

The group $\Gamma$ acts on the projective cubic $\bar{V}_{t}$ by rational maps. It has a linear subgroup isomorphic to $S_{4}$ (this subgroup is generated by the group of sign changes and the elements $\alpha_{*} \gamma_{*}$ and $\beta_{*}$ of section 2). This linear subgroup acts on $\bar{V}_{t}$ by morphisms (biholomorphic maps) and therefore permutes the 27 lines. The whole of $\Gamma$ is generated by this linear subgroup and the involution $\gamma_{*}$. This latter involution acts on $\bar{V}_{t}$ by the rational map which blows up the intersection point of the lines $x=0$ and $y=0$ at infinity and blows down the line $z=0$ at infinity. It is not hard to derive the action on homology also from this point of view. 


\section{The four-holed sphere}

Suppose $N$ is a four-holed sphere. Its fundamental group $\pi$ is freely generated by three peripheral elements $\delta_{1}, \delta_{2}, \delta_{3}$. The generator $\delta_{4}$ of the fourth boundary component satisfies the relation

$$
\delta_{1} \delta_{2} \delta_{3} \delta_{4}=1
$$

and we consider the redundant geometric presentation

$$
\left\langle X, Y, Z, \delta_{1}, \delta_{2}, \delta_{3}, \delta_{4} \mid \delta_{1} \delta_{2} \delta_{3} \delta_{4}=1, X=\delta_{1} \delta_{2}, Y=\delta_{2} \delta_{3}, Z=\delta_{3} \delta_{1}\right\rangle .
$$

Let $t=\left(t_{1}, t_{2}, t_{3}, t_{4}\right) \in \mathbb{C}^{4}$. The relative character variety results by fixing the trace

$$
\operatorname{tr} \rho\left(\delta_{i}\right)=t_{i}
$$

for $i=1,2,3,4$. In that case (see Goldman [6, 5], Benedetto-Goldman [1], which is based on Magnus [10]), the relative character variety is given by the cubic hypersurface in $\mathbb{C}^{3}$ defined by:

$$
\begin{aligned}
x^{2}+y^{2}+z^{2}+x y z= & \left(t_{1} t_{2}+t_{3} t_{4}\right) x+\left(t_{1} t_{4}+t_{2} t_{3}\right) y+\left(t_{1} t_{3}+t_{2} t_{4}\right) z \\
& +\left(4-t_{1}^{2}-t_{2}^{2}-t_{3}^{2}-t_{4}^{2}-t_{1} t_{2} t_{3} t_{4}\right) .
\end{aligned}
$$

Using the change of coordinates that reverses the signs of $x, y$ and $z$ puts this in the form:

$$
\kappa_{P, Q, R}(x, y, z)=S
$$

where

$$
\kappa_{P, Q, R}(x, y, z)=x^{2}+y^{2}+z^{2}-x y z-P x-Q y-R z-2
$$

as in the Introduction, and $P=-\left(t_{1} t_{2}+t_{3} t_{4}\right), Q=-\left(t_{1} t_{4}+t_{2} t_{3}\right), R=-\left(t_{1} t_{3}+\right.$ $\left.t_{2} t_{4}\right)$, and $S=\left(2-t_{1}^{2}-t_{2}^{2}-t_{3}^{2}-t_{4}^{2}-t_{1} t_{2} t_{3} t_{4}\right)$.

We first discuss the the group Aut $\left(\kappa_{P, Q, R}\right)$ of polynomial automorphisms of the polynomial $\kappa_{P, Q, R}$.

Any affine automorphism $\phi=\left(\phi_{1}, \phi_{2}, \phi_{3}\right)$ of $\mathbb{C}^{3}$ that preserves $\kappa_{P, Q, R}$ extends to $\mathbb{P}^{3}(\mathbb{C})$ and must therefore preserve the intersection of the closure $\bar{V}_{t}$ of the fiber $V_{t}=\kappa_{P, Q, R}^{-1}(t)$ of $\kappa_{P, Q, R}$ with the plane at infinity. This intersection consists of the three coordinate lines. It follows that $\phi$ must permute the coordinates $x, y, z$ up to multiplication by scalars. Inspecting $\kappa_{P, Q, R}$ we see that the only nontrivial multiplication by scalars that can occur is multiplying two coordinates by -1 if the corresponding two of $P, Q, R$ are zero. We thus see:

Lemma 5.1. The group $L_{P, Q, R}=\operatorname{Aut}\left(\kappa_{P, Q, R}\right) \cap \operatorname{Aff}\left(\mathbb{C}^{3}\right)$ is generated by permutation and sign-change automorphisms and is one of

$$
\{1\}, \quad C_{2}, \quad C_{2} \times C_{2}, \quad S_{3}, \quad S_{4},
$$

according as no two of $P, Q, R$ are equal; just two of them are equal but nonzero; just two of them are equal to zero; all three equal and nonzero; all three zero. 
Direct substitution shows the following three involutions are in $\operatorname{Aut}\left(\kappa_{P, Q, R}\right)$ :

$$
\begin{aligned}
& \tau_{1}:(x, y, z) \mapsto(x, y, x y-z+R) \\
& \tau_{2}:(x, y, z) \mapsto(y z-x+P, y, z) \\
& \tau_{3}:(x, y, z) \mapsto(x, x z-y+Q, z) .
\end{aligned}
$$

Theorem 5.2. Aut $\left(\kappa_{P, Q, R}\right)$ is generated by the above three involutions and $L_{P, Q, R}$. The three involutions generate a normal subgroup which is the free product $C_{2} * C_{2} * C_{2}=\left\langle\tau_{1}, \tau_{2}, \tau_{3} \mid \tau_{1}^{2}=\tau_{2}^{2}=\tau_{3}^{2}=1\right\rangle$ and

$$
\operatorname{Aut}\left(\kappa_{P, Q, R}\right)=\left(C_{2} * C_{2} * C_{2}\right) \rtimes L_{P, Q, R} .
$$

(We identify this $C_{2} * C_{2} * C_{2}$ with $\mathrm{PGL}(2, \mathbb{Z})_{(2)}$ in Theorem 5.4.)

Proof. The proof follows Horowitz's proof [9] for the case $P=Q=R=0$. The group generated by the $\tau_{i}$ is normalized by $L_{P, Q, R}$. Let $\phi=\left(\phi_{1}, \phi_{2}, \phi_{3}\right) \in$ $\operatorname{Aut}\left(\kappa_{P, Q, R}\right)$ have degree $>1$. We claim there is a unique one of the three involutions $\tau_{i}$ for which $\tau_{i} \circ \phi$ has lower degree than $\phi$. It then follows that there is a unique reduced word $\omega$ in the $\tau_{i}$ such that $\omega \circ \phi \in L_{P, Q, R}$. This implies the theorem.

To prove the claim, assume for convenience that $\operatorname{deg} \phi_{1} \leq \operatorname{deg} \phi_{2} \leq \operatorname{deg} \phi_{3}$ and $\operatorname{deg} \phi_{3}>1$. The highest order terms in the equation

$\phi_{1}^{2}+\phi_{2}^{2}+\phi_{3}^{2}-\phi_{1} \phi_{2} \phi_{3}-P \phi_{1}-Q \phi_{2}-R \phi_{3}=x^{2}+y^{2}+z^{2}-x y z-P x-Q y-R z$

imply that $\operatorname{deg} \phi_{3}=\operatorname{deg} \phi_{1}+\operatorname{deg} \phi_{2}$ and that the highest order terms in $\phi_{1} \phi_{2}-\phi_{3}$ must cancel. It follows that $\tau_{1} \circ \phi$ has lower degree than $\phi$. Clearly, neither of the other $\tau_{i}$ has this property.

We now discuss the mapping class group $\pi_{0} \operatorname{Homeo}\left(N, \partial_{1} N, \ldots, \partial_{4} N\right)$ which fixes the boundary components of the four-holed sphere $N$.

We can array the punctures of $N$ in order around the equator of a 2 -sphere and place our basepoint on the part of the equator joining the first two punctures. Then the involution $T_{1}$ that reflects north-south across the equator takes the elements $X=\delta_{1} \delta_{2}, Y=\delta_{2} \delta_{3}$, and $Z=\delta_{3} \delta_{1}$ of $\pi_{1} N$ to $\delta_{1}^{-1} \delta_{2}^{-1}=\delta_{1}^{-1} X^{-1} \delta_{1}$, $\delta_{3}^{-1} \delta_{2}^{-1}=Y^{-1}$, and $\delta_{2} \delta_{4}$. The action on traces is $(x, y, z) \mapsto(x, y, x y-z+R)$. Similarly, by arraying the four punctures in the orders 1324 and 1243 around the equator we get two more involutions $T_{2}, T_{3}$; the actions on $\mathbb{C}^{3}$ of these three involutions are the three involutions $\tau_{1}, \tau_{2}, \tau_{3}$ above.

It is not hard to see that the pairwise products $T_{3} T_{1}, T_{1} T_{2}$, and $T_{2} T_{3}$ of these involutions are the Dehn twists $T_{X}, T_{Y}, T_{Z}$, on the three separating simple closed curves that represent the elements $X, Y, Z$ of $\pi_{1} N$. It is well-known that any two of these Dehn twists freely generate $\pi_{0} \mathrm{Homeo}^{+}\left(N, \partial_{1} N, \ldots, \partial_{4} N\right)$. It follows that $T_{1}, T_{2}, T_{3}$ generate $\pi_{0} \operatorname{Homeo}\left(N, \partial_{1} N, \ldots, \partial_{4} N\right)$. Moreover, they generate it as a free product $C_{2} * C_{2} * C_{2}$ since their action on $\mathbb{C}^{3}$ is as this free product. Summarizing: 
Theorem 5.3. The mapping class group $\pi_{0} \operatorname{Homeo}\left(N, \partial_{1} N, \ldots, \partial_{4} N\right)$ is the free product $C_{2} * C_{2} * C_{2}$ generated by the three involutions $T_{1}, T_{2}, T_{3}$ above, and it acts on the relative character varieties via the action of $C_{2} * C_{2} * C_{2}$ of the previous theorem.

Note that our computation implies that the Dehn twists $T_{X}$ and $T_{Y}$ act on the relative character varieties by the following actions on $\mathbb{C}^{3}$

$$
\begin{aligned}
& \tau_{X}:(x, y, z) \mapsto\left(x, x^{2} y-x z+R x-y+Q, x y-z+R\right) \\
& \tau_{Y}:(x, y, z) \mapsto\left(y z-x+P, y, y^{2} z-x y+P y-z+R\right) .
\end{aligned}
$$

This agrees with the calculation in [6] (except for sign differences because we changed coordinates to reverse the signs of $x, y, z)$. But it should not agree, since our Dehn twists are actually the inverses of the Dehn twists of [6]. The discrepancy is because automorphisms $\gamma$ of $\pi_{1} N$ act on characters $\chi$ by $(\gamma, \chi) \mapsto$ $\gamma \chi:=\chi \circ \gamma^{-1}$, but [6] uses $(\gamma, \chi) \mapsto \chi \circ \gamma$, which gives a right-action.

Theorem 5.4. There are natural geometric isomorphisms:

$$
\begin{aligned}
\pi_{0} \operatorname{Homeo}\left(N, \partial_{1} N, \partial N-\partial_{1} N\right) & \cong \operatorname{PGL}(2, \mathbb{Z}) \\
\pi_{0} \operatorname{Homeo}\left(N, \partial_{1} N, \ldots, \partial_{4} N\right) & \cong \operatorname{PGL}(2, \mathbb{Z})_{(2)} \\
\pi_{0} \operatorname{Homeo}\left(N, \partial_{N}\right) & \cong \operatorname{PGL}(2, \mathbb{Z})_{(2)} \rtimes S_{4} \\
& \cong \operatorname{PGL}(2, \mathbb{Z}) \ltimes \Sigma \cong \Gamma
\end{aligned}
$$

Proof. This is mostly well-known, so we describe it briefly. $\mathrm{GL}(2, \mathbb{Z})$ acts on $T^{2}=\mathbb{R}^{2} / \mathbb{Z}^{2}$ by linear maps, giving an isomorphism of $\mathrm{GL}(2, \mathbb{Z})$ with the mapping class group of $T^{2}$. The central element $-I \in \mathrm{GL}(2, \mathbb{Z})$ acts as an involution fixing the four half-integer points

$$
(0,0), \quad\left(\frac{1}{2}, 0\right), \quad\left(0, \frac{1}{2}\right), \quad\left(\frac{1}{2}, \frac{1}{2}\right),
$$

so the quotient $\operatorname{PGL}(2, \mathbb{Z})=\operatorname{SL}(2, \mathbb{Z}) /\{ \pm I\}$ acts on the orbit space, which is $S^{2}$, and permutes the four branch points. This action fixes the first branch point and permutes the other three via the quotient $\operatorname{PGL}(\mathbb{Z} / 2,2)$ (isomorphic to $S_{3}$ ). Thus the kernel

$$
\operatorname{PGL}(2, \mathbb{Z})_{(2)}=\operatorname{Ker}(\operatorname{PGL}(\mathbb{Z}, 2) \rightarrow \operatorname{PGL}(\mathbb{Z} / 2,2))
$$

acts on the 2 -sphere fixing all four branch-points. By replacing each branch point by the circle of tangent directions at the point, we get actions of $\operatorname{PGL}(2, \mathbb{Z})$ and $\operatorname{PGL}(2, \mathbb{Z})_{(2)}$ on the four-holed sphere $N$. Since a mapping class of the four-holed sphere gives a mapping class of the four-pointed sphere, which then lifts, up to $C_{2}$ ambiguity, to a mapping class on the torus and thence to an action on its homology, this gives the first two isomorphisms of the theorem.

Finally, the mapping class group $\pi_{0} \operatorname{Homeo}(N, \partial N)$ acts on the set of boundary components, giving a homomorphism to $S_{4}$, determining it as an $S_{4}$ extension of $\operatorname{PGL}(2, \mathbb{Z})_{(2)}=\pi_{0} \operatorname{Homeo}\left(N, \partial_{1} N, \ldots, \partial_{4} N\right)$ :

$$
1 \rightarrow \operatorname{PGL}(2, \mathbb{Z})_{(2)} \rightarrow \pi_{0} \operatorname{Homeo}(N, \partial N) \rightarrow S_{4} \rightarrow 1
$$


This is a split extension, since we can map $S_{4}$ to $\pi_{0} \operatorname{Homeo}(N, \partial N)$ as follows: Construct $N$ from the boundary of a regular tetrahedron by removing neighborhoods of the vertices; the tetrahedral group $S_{4}$ then acts by rigid motions on $N$. The Klein four-group $\Sigma \subset S_{4} \subset \pi_{0} \operatorname{Homeo}(N, \partial N)$ is the kernel of the action of $\pi_{0} \operatorname{Homeo}(N, \partial N)$ on homology of the torus $T_{2}$ discussed above: each element of the Klein four-group comes from a translation of $T^{2}=\mathbb{R}^{2} / \mathbb{Z}^{2}$ by an element of the half-integer lattice, and these act trivially on homology. Summarizing, we see that $\pi_{0} \operatorname{Homeo}(N, \partial N)$ fits in place of $\Gamma$ in a diagram identical to diagram (1) of the Introduction. In fact, both diagrams represent the group in the middle as the pullback for the diagram

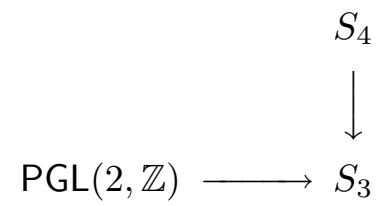

so they are isomorphic, and $\pi_{0} \operatorname{Homeo}(N, \partial N) \cong \Gamma$.

By explicitly lifting the above generating involutions $T_{1}, T_{2}, T_{3}$ to the torus and computing action on homology one finds that they correspond under this isomorphism to the elements

$$
\left(\begin{array}{cc}
1 & 0 \\
0 & -1
\end{array}\right), \quad\left(\begin{array}{cc}
1 & 0 \\
2 & -1
\end{array}\right), \quad\left(\begin{array}{cc}
1 & 2 \\
0 & -1
\end{array}\right)
$$

respectively. The three Dehn twists $T_{X}=T_{3} T_{1}, T_{Y}=T_{1} T_{2}, T_{Z}=T_{2} T_{3}$, therefore correspond to

$$
\left(\begin{array}{ll}
1 & 2 \\
0 & 1
\end{array}\right), \quad\left(\begin{array}{cc}
1 & 0 \\
-2 & 1
\end{array}\right), \quad\left(\begin{array}{ll}
1 & -2 \\
2 & -3
\end{array}\right)
$$

We now return to the topology of the fibers of the polynomials $\kappa_{P, Q, R}$.

Theorem 5.5. Each fiber $V_{t}$ of $\kappa_{P, Q, R}$ has at most isolated singularities. It has reduced homology only in dimension 2 , and $H_{2}\left(V_{t}\right)=H_{2}(V) / I\left(V_{t}\right)$, where $V$ is a typical non-singular fiber and $I\left(V_{t}\right)$ is the "subgroup of vanishing cycles" for the singularities of $V_{t}$, which is non-trivial if and only if $V_{t}$ is singular. Moreover, $H_{2}(V) \cong \mathbb{Z}^{5}$, and it is the direct sum of the groups of vanishing cycles $I\left(V_{t}\right)$, summed over the singular fibers of $\kappa_{P, Q, R}$ (in particular, there are at most five singular fibers).

Proof. Since the gradient $d \kappa_{P, Q, R}$ differs from the gradient $d \kappa$ by the constant vector $(P, Q, R)$, Lemma 1.1 applies to $\kappa_{P, Q, R}$, so $\kappa_{P, Q, R}$ is tame in Broughton's sense. Broughton [2] (see also [12]) proves that tameness implies the statements of the theorem, except, of course, for the fact that $H_{2}(V) \cong \mathbb{Z}^{5}$. We will see this below, where we show that each non-singular fiber $V$ of $\kappa_{P, Q, R}$ has the same topology as the non-singular fibers of $\kappa$.

That $\kappa_{P, Q, R}$ has at most five singular fibers is also easy to see by direct computation, and moreover, that for generic $P, Q, R$ it has exactly five singular fibers, 
each with a single quadratic singularity. However, more complex singularities can occur. Benedetto and Goldman discuss this topology in [1], and describe, in particular, under what conditions a relative character variety for $N$ can be a singular fiber of the relevant cubic $\kappa_{P, Q, R}$.

In any case, we restrict now to a nonsingular fiber $V=\kappa_{P, Q, R}^{-1}(t)$ of some $\kappa_{P, Q, R}$. Denote by $\bar{V}$ the closure of $V$ in $\mathbb{P}^{3}(\mathbb{C})$. As discussed in sections 3 and 4 for the case $P=Q=R=0, \bar{V}$ is a nonsingular projective cubic which intersects the projective plane at infinity in the three lines $x=0, y=0, z=0$, and these are three of the 27 lines on the cubic surface $\bar{V}$, the others arising through singular fibers of the projection of $V$ to the three coordinate axes. The computations are essentially the same as the case $P=Q=R=0$. Thus the topology of the nonsingular cubic $V$ does not change as the parameters $P, Q, R, t$ that define it change. The action of the automorphism group of this cubic on homology, which is always a subgroup of the group $\Gamma$, will therefore also not change, so it is as described in the Introduction.

\section{References}

[1] R. Benedetto and W. M. Goldman, The topology of the relative character variety of the quadruply-punctured sphere, Experimental Math. 8 (1999), 85-103.

[2] S. A. Broughton, Milnor number and the topology of polynomial hypersurfaces, Inv. Math. 92 (1988), 217-241.

[3] S. Cappell, R. Lee, and E. Y. Miller, The Torelli group action on representation spaces, Contemp. Math. 258 (2000), 47-70.

[4] - The action of the Torelli group on the homology of representation spaces is nontrivial, Topology 39 (2000), 851-871.

[5] R. Fricke, Über die Theorie der automorphen Modulgruppen, Nachr. Akad. Wiss. Göttingen (1896), 91-101.

[6] W. M. Goldman, Ergodic Theory on Moduli Spaces, Ann. Math. 146 (1997), 1-33.

[7] Action of the modular group on real SL(2)-characters of a one-holed torus, Topology and Geometry 7 (2003), 443-486.

[8] _ An exposition of results of Fricke, (in preparation).

[9] R. D. Horowitz, Induced automorphisms on Fricke characters of free groups, Trans. Amer. Math. Soc. 208 (1975), 41-50.

[10] W. Magnus, Rings of Fricke characters and automorphism groups of free groups, Math. Zeit. 170 (1980), 91-103.

[11] W. D. Neumann, A calculus for plumbing applied to the topology of complex surface singularities and degenerating complex curves, Trans. Amer. Math. Soc. 268 (1981), 299343.

[12] W. D. Neumann and P. Norbury, Unfolding singularities at infinity, Math. Ann. 318 (2000), 149-180.

[13] J. Nielsen, Die Isomorphismen der allgemeinen unendlichen Gruppe mit zwei Erzeugenden, Math. Ann. 71 (1918), 385-397.

Department of Mathematics, The University of Maryland, College Park, MD 20742

E-mail address: wmg@math.umd.edu

Department of Mathematics, Barnard College, Columbia University, New York, NY 10027

E-mail address: neumann@math.columbia.edu 